\title{
Concircular Curvature on Warped Product Manifolds and Applications
}

\author{
Uday Chand $\mathrm{De}^{1} \cdot$ Sameh Shenawy ${ }^{2} \cdot$ Bülent Ünal $^{3}$ \\ Received: 26 February 2019 / Revised: 21 November 2019 \\ (c) Malaysian Mathematical Sciences Society and Penerbit Universiti Sains Malaysia 2019
}

\begin{abstract}
This study aims mainly at investigating the effects of concircular flatness and concircular symmetry of a warped product manifold on its fiber and base manifolds. Concircularly flat and concircularly symmetric warped product manifolds are investigated. The divergence-free concircular curvature tensor on warped product manifolds is considered. Finally, we apply some of these results to generalized Robertson-Walker and standard static space-times.
\end{abstract}

Keywords Concircular curvature · Concircularly symmetric manifolds · Concircularly flat manifolds · Warped product manifolds

Mathematics Subject Classification Primary 53C21 - 53C25; Secondary 53C50 . $53 \mathrm{C} 80$

\section{Introduction}

A transformation which preserves geodesic circles is called a concircular transformation [31]. The geometry which deals with concircular transformation is called

Communicated by Rosihan M. Ali.

$\triangle$ Uday Chand De

uc_de@yahoo.com

Sameh Shenawy

drssshenawy@eng.modern-academy.edu.eg; drshenawy@mail.com

Bülent Ünal

bulentunal@mail.com

1 Department of Pure Mathematics, University of Calcutta, 35 Bally-Gaunge Circular Road, Kolkata, West Bengal 700019, India

2 Basic Science Department, Modern Academy for Engineering and Technology, Maadi, Egypt

3 Department of Mathematics, Bilkent University, 06800 Bilkent, Ankara, Turkey 
concircular geometry. The concircular curvature tensor $\mathcal{C}$ remains invariant under concircular transformation of a (pseudo-)Riemannian manifold $M$. $M$ is called concircularly flat if its concircular curvature tensor $\mathcal{C}$ vanishes at every point. A concircularly flat manifold $M$ is a manifold of constant curvature. Thus, the tensor $\mathcal{C}$ measures the deviation of $M$ from constant curvature. (For further details, see [1,31].)

In a series of studies, Pokhariyal and Mishra studied the recurrent properties and relativistic significance of concircular curvature tensor, among many others, in Riemannian manifolds [22-25]. Concircularly semi-symmetric $K$-contact manifolds are considered in [18], and concircularly recurrent Finsler manifolds are studied in [33]. In [19], the authors considered $N(k)$-contact metric manifolds satisfying $\mathcal{C} \cdot \mathcal{P}=0$, where $\mathcal{P}$ denotes the projective curvature tensor. Similarly, a study of $(k, \mu, v)$-contact metric 3-manifolds satisfying one of the conditions $\nabla \mathcal{C}=0, \mathcal{C}(\zeta, X) \cdot \mathcal{C}=0$, $\mathcal{R}(\zeta, X) \cdot \mathcal{C}=0$, where $\zeta$ is the Reeb field, is considered in [16]. Perfect fluid space-times with either vanishing or divergence-free concircular curvature tensor are considered in [2]. The authors of [34] considered equitorsion concircular mapping between generalized Riemannian manifolds (in the sense of Eisenhart's definition) and obtained some invariant curvature tensors. These tensors are generalizations of concircular curvature tensor on Riemannian manifolds. In a recent paper [10], Chen provided some classification of Ricci solitons with respect to a concircular potential field. In [17], the concept of special concircular vector fields is introduced and it is proved that an $n$-dimensional Riemannian manifold that admits $n$ linearly independent special concircular vector fields has constant sectional curvature. Similarly, in [9], the authors characterize the local structure of a Riemannian manifold whose Codazzi tensor has exactly two distinct eigenvalues. In [21], it is proven that each concircularly recurrent manifold is necessarily a recurrent manifold.

Motivated by these studies and many others, the main purpose of this article is to study concircular curvature tensor on warped product manifolds and to apply some of the results to two different $n$-dimensional space-times, namely, generalized Robertson-Walker space-times and standard static space-times. Concircularly flat and concircularly symmetric warped product manifolds are also considered. Finally, divergence-free concircular curvature tensor on warped product manifolds is investigated.

This article is organized as follows. The next section presents the main properties of the concircular curvature tensor. In Sect. 3, the semi-symmetries of the concircular curvature tensor are investigated. Section 4 is devoted to the study of concircularly flat warped product manifolds, whereas Sect. 5 is devoted to the study of concircularly symmetric warped product manifolds. Finally, divergence-free concircular curvature tensor on warped product space-time models is considered in Sect. 6.

\section{Concircular Curvature Tensor}

Let $(M, g)$ be a pseudo-Riemannian $n$-dimensional manifold $n \geq 3$. Throughout this section, $\nabla, \mathcal{R}$, Ric and $\tau$ denote the Levi-Civita connection, curvature tensor, Ricci curvature and scalar curvature of the metric tensor $g$, respectively. 
The concircular curvature tensor $\mathcal{C}$ on a pseudo-Riemannian manifold $(M, g, \nabla)$ is defined as follows $[2,25,28,32]$. Let $X, Y, Z, V \in \mathfrak{X}(M)$, then

$$
\begin{aligned}
\mathcal{C}(X, Y) Z= & \mathcal{R}(X, Y) Z \\
& -\frac{\tau}{n(n-1)}[g(X, Z) Y-g(Y, Z) X],
\end{aligned}
$$

where $\mathcal{R}(X, Y) Z=\nabla_{Y} \nabla_{X} Z-\nabla_{X} \nabla_{Y} Z+\nabla_{[X, Y]} Z$ is the Riemann curvature tensor. It is clear that $\mathcal{C}(X, Y) Z$ is skew-symmetric in the first two indices. Furthermore,

$$
\begin{aligned}
\mathcal{C}(X, Y, Z, V)= & \mathcal{R}(X, Y, Z, V) \\
& -\frac{\tau}{n(n-1)}[g(X, Z) g(Y, V)-g(Y, Z) g(X, V)] .
\end{aligned}
$$

The definition of the concircular curvature tensor in local coordinates is as follows

$$
\mathcal{C}_{i j k l}=\mathcal{R}_{i j k l}-\frac{\tau}{n(n-1)}\left[g_{i k} g_{j l}-g_{j k} g_{i l}\right],
$$

where $\tau=g^{i j} \mathcal{R}_{i j}$ is the scalar curvature. This formula suggests a generalization of this tensor of the form

$$
\mathcal{K}_{i j k l}=a_{0} \mathcal{R}_{i j k l}+a_{1} g_{i j} g_{k l}+a_{2} g_{i k} g_{j l}+a_{3} g_{j k} g_{i l},
$$

where $a_{i}$ are constants and $a_{0} \neq 0$. Assume that a pseudo-Riemannian manifold $(M, g)$ is a $\mathcal{K}$-curvature flat manifold, then

$$
a_{0} \mathcal{R}_{i j k l}+a_{1} g_{i j} g_{k l}+a_{2} g_{i k} g_{j l}+a_{3} g_{j k} g_{i l}=0
$$

Multiplying both sides by $g^{i l}$, we get

$$
-a_{0} \mathcal{R}_{j k}+a_{1} g_{k j}+a_{2} g_{k j}+n a_{3} g_{j k}=0
$$

Again, by multiplying both sides of Eq. (2.5) by $g^{i k}$, we get

$$
a_{0} \mathcal{R}_{j l}+a_{1} g_{j l}+n a_{2} g_{j l}+a_{3} g_{j l}=0
$$

Thus $(M, g)$ is Einstein with

$$
\begin{aligned}
& \mathcal{R}_{j k}=\frac{a_{1}+a_{2}+n a_{3}}{a_{0}} g_{j k} \\
& \mathcal{R}_{j l}=-\left(\frac{a_{1}+n a_{2}+a_{3}}{a_{0}}\right) g_{j l} .
\end{aligned}
$$

Consequently, a second contraction implies

$$
a_{1}+a_{2}+n a_{3}=\frac{\tau}{n} a_{0}
$$




$$
a_{1}+n a_{2}+a_{3}=-\frac{\tau}{n} a_{0}
$$

However, Eq. (2.5) yields

$$
n a_{1}+a_{2}+a_{3}=0 .
$$

These equations imply that

$$
a_{1}=0, \quad a_{2}=-a_{3}=\frac{-a_{0} \tau}{n(n-1)}
$$

Again, Eq. (2.5) becomes

$$
a_{0}\left[\mathcal{R}_{i j k l}-\frac{\tau}{n(n-1)}\left(g_{i k} g_{j l}-g_{j k} g_{i l}\right)\right]=0 .
$$

Thus, $M$ is of constant sectional curvature. Therefore, the only $\mathcal{K}$-curvature flat tensor is the concircular curvature tensor and we have:

Theorem 1 Let $M$ be a $\mathcal{K}$-curvature flat manifold. Then $M$ is of constant curvature and

$$
a_{1}=0, \quad a_{2}=-a_{3}=\frac{-a_{0} \tau}{n(n-1)}
$$

i.e., $\mathcal{K}$ is a constant multiple of $\mathcal{C}$.

This result is proved in [31] when $\mathcal{K}=\mathcal{C}$. Moreover, it is found in [33] for the Finslerian case. Assume that $M$ is a 4-dimensional space-time obeying Einstein's field equation with cosmological constant, i.e.,

$$
\mathcal{R}_{i j}-\frac{\tau}{2} g_{i j}+\Lambda g_{i j}=k T_{i j}
$$

where $T$ is the energy-momentum tensor. Let us define

$$
\begin{aligned}
\mathcal{K}_{j l} & =g^{i k} \mathcal{K}_{i j k l} \\
& =a_{0} \mathcal{R}_{j l}+\left(a_{1}+n a_{2}+a_{3}\right) g_{j l} .
\end{aligned}
$$

Now Eq. (2.7) becomes

$$
\mathcal{K}_{i j}-\left(a_{1}+n a_{2}+a_{3}\right) g_{i j}-\frac{a_{0} \tau}{2} g_{i j}+a_{0} \Lambda g_{i j}=a_{0} k T_{i j} .
$$

Thus

$$
\nabla_{i} \mathcal{K}_{j}^{i}-\frac{a_{0}}{2} \nabla_{j} \tau=a_{0} k \nabla_{i} T_{j}^{i}
$$

We can now state: 
Theorem 2 In a relativistic space-time obeying Einstein's field equations, the energymomentum tensor is divergence free if and only if

$$
\nabla_{i} \mathcal{K}_{j}^{i}=\frac{a_{0}}{2} \nabla_{j} \tau
$$

\section{Semi-symmetries of $\mathcal{C}$}

It is noted that Eq. (2.2) has the form

$$
\begin{aligned}
\mathcal{C} & =\mathcal{R}-\frac{\tau}{2 n(n-1)}(g \wedge g) \\
& =\mathcal{R}-\frac{\tau}{n(n-1)} \mathcal{G},
\end{aligned}
$$

where $\wedge$ is Kulkarni-Nomizu product of two symmetric 2-tensors (see [6, p. 47]) and $\mathcal{G}=\frac{1}{2}(g \wedge g)$. This equation leads us to

$$
\begin{aligned}
\mathcal{R} \cdot \mathcal{C} & =\mathcal{R} \cdot \mathcal{R}-\frac{\tau}{n(n-1)} \mathcal{R} \cdot \mathcal{G} \\
& =\mathcal{R} \cdot \mathcal{R},
\end{aligned}
$$

where $\mathcal{R} \cdot \mathcal{C}$ means that $\mathcal{R}(X, Y)$ acts as a derivation on $\mathcal{C}$ for any vector fields $X, Y \in$ $\mathfrak{X}(M)$. However,

$$
\begin{aligned}
\mathcal{C} \cdot \mathcal{R} & =\left(\mathcal{R}-\frac{\tau}{n(n-1)} \mathcal{G}\right) \cdot \mathcal{R} \\
& =\mathcal{R} \cdot \mathcal{R}-\frac{\tau}{n(n-1)} \mathcal{G} \cdot \mathcal{R}
\end{aligned}
$$

We thus have the following:

Proposition 1 A pseudo-Riemannian manifold $M$ admits a semi-symmetric concircular curvature tensor $\mathcal{C}$ if and only if $M$ is semi-symmetric.

Proposition 2 A pseudo-Riemannian manifold $M$ is pseudo-symmetric (i.e., $\mathcal{R} \cdot \mathcal{R}=$ $\left.\frac{\tau}{n(n-1)} \mathcal{G} \cdot \mathcal{R}\right)$ if and only if $\mathcal{C} \cdot \mathcal{R}=0$.

On the other hand

$$
\begin{aligned}
\mathcal{C} \cdot \mathcal{C} & =\left(\mathcal{R}-\frac{\tau}{n(n-1)} \mathcal{G}\right) \cdot\left(\mathcal{R}-\frac{\tau}{n(n-1)} \mathcal{G}\right) \\
& =\mathcal{R} \cdot \mathcal{R}-\frac{\tau}{n(n-1)} \mathcal{G} \cdot \mathcal{R} .
\end{aligned}
$$

We thus have: 
Proposition 3 A pseudo-Riemannian manifold $M$ is pseudo-symmetric if and only if $\mathcal{C} \cdot \mathcal{C}=0$.

Now, assume that $\mathcal{C}$ vanishes on $M$. Then

$$
\mathcal{R}=\frac{\tau}{n(n-1)} \mathcal{G}
$$

i.e., $M$ is of constant curvature $\kappa=\frac{\tau}{n(n-1)}$. The converse is also true and we have:

Proposition 4 A concircularly flat pseudo-Riemannian manifold $M$ (i.e., $M$ admits a flat concircular curvature tensor) is of constant curvature.

A pseudo-Riemannian manifold $M$ is said to be concircularly symmetric if $\nabla \mathcal{C}=0$. It is clear that

$$
\nabla \mathcal{C}=\nabla \mathcal{R}-\frac{1}{n(n-1)}(\nabla \tau) \mathcal{G} .
$$

Assume that $M$ is concircularly symmetric i.e., $\nabla \mathcal{C}=0$. Then

$$
\nabla \mathcal{R}=\frac{1}{n(n-1)}(\nabla \tau) \mathcal{G} .
$$

The second Bianchi identity implies that $M$ is of constant curvature $\kappa$ and consequently $M$ is locally symmetric. Conversely, now suppose that $M$ is locally symmetric, that is, $\nabla \mathcal{R}=0$, then the scalar curvature is constant and hence $\nabla \mathcal{C}=0$. This discussion leads to the following result.

Proposition 5 A pseudo-Riemannian manifold $(M, g)$ is locally symmetric if and only if it is concircularly symmetric.

In [8], it is proved that a semi-symmetric manifold $(M, g)$ whose Ricci tensor is a Codazzi tensor is a locally symmetric manifold. This result and Proposition (5) lead to the following.

Corollary 1 A semi-symmetric manifold $(M, g)$ whose Ricci tensor is a Codazzi tensor is a concircularly symmetric manifold.

\section{Concircularly Flat Warped Products}

In this section, we shall first give some basic definitions about warped product manifolds and then apply them to study the concircularly flat warped products. Suppose that $\left(M_{1}, g_{1}, \nabla^{1}\right)$ and $\left(M_{2}, g_{2}, \nabla^{2}\right)$ are two smooth pseudo-Riemannian manifolds equipped with Riemannian metrics $g_{i}$, where $\nabla^{i}$ is the Levi-Civita connection of the metric $g_{i}$ for $i=1,2$. Further suppose that $\pi_{1}: M_{1} \times M_{2} \rightarrow M_{1}$ and $\pi_{2}: M_{1} \times M_{2} \rightarrow M_{2}$ are the natural projection maps of the Cartesian product $M_{1} \times M_{2}$ onto $M_{1}$ and $M_{2}$, respectively. If $f: M_{1} \rightarrow(0, \infty)$ is a positive real-valued 
smooth function, then the warped product manifold $M_{1} \times{ }_{f} M_{2}$ is the product manifold $M_{1} \times M_{2}$ equipped with the metric tensor $g=g_{1} \oplus f^{2} g_{2}$ defined by

$$
g=\pi_{1}^{*}\left(g_{1}\right) \oplus\left(f \circ \pi_{1}\right)^{2} \pi_{2}^{*}\left(g_{2}\right),
$$

where ${ }^{*}$ denotes the pull-back operator on tensors $[7,20,30]$. The function $f$ is called the warping function of the warped product manifold $M_{1} \times{ }_{f} M_{2}$. In particular, if $f=1$, then $M_{1} \times{ }_{1} M_{2}=M_{1} \times M_{2}$ is the usual Cartesian product manifold. It is clear that the submanifold $M_{1} \times\{q\}$ is isometric to $M_{1}$ for every $q \in M_{2}$. Moreover, $\{p\} \times M_{2}$ is homothetic to $M_{2}$. Throughout this article we use the same notation for a vector field and for its lift to the product manifold $[11,12,29,30]$.

Throughout this section, $(M, g, \nabla)$ is a (singly) warped product manifold of $\left(M_{i}, g_{i}, \nabla^{i}\right), i=1,2$ with dimensions $n_{i} \neq 1$, where $n=n_{1}+n_{2} \cdot \mathcal{R}, \mathcal{R}^{i}$ and Ric, $\operatorname{Ric}^{i}$ denote the curvature tensor and Ricci curvature tensor on $M, M_{i}$, respectively. Moreover, $\operatorname{grad} f, \Delta f$ denote gradient and Laplacian of $f$ on $M_{1}$ and $f^{\sharp}=f \Delta f+\left(n_{2}-1\right) g_{1}(\operatorname{grad} f, \operatorname{grad} f)$. Finally, concircular curvature tensor on $M$ and $M_{i}$ is denoted by $\mathcal{C}$ and $\mathcal{C}^{i}$, respectively.

We now define generalized Robertson-Walker space-times. Let $(M, g)$ be an $n$ dimensional pseudo-Riemannian manifold and $f$ be a positive smooth function on an open connected subinterval $I$ of $\mathbb{R}$. Then the $(n+1)$-dimensional product manifold $I \times M$ furnished with the metric tensor

$$
\bar{g}=-\mathrm{d} t^{2} \oplus f^{2} g
$$

is called a generalized Robertson-Walker space-time and is denoted by $\bar{M}=I \times{ }_{f} M$, where $\mathrm{d} t^{2}$ is the Euclidean usual metric tensor on $I$. These space-times are generalization of the well-known Robertson-Walker space-times [15,26,27]. From now on, we will denote $\frac{\partial}{\partial t} \in \mathfrak{X}(I)$ by $\partial_{t}$ to state our results in simpler forms.

Similarly, we define standard static space-times. Let $(M, g)$ be an $n$-dimensional pseudo-Riemannian manifold and $f: M \rightarrow(0, \infty)$ be a smooth function. Then the $(n+1)$-dimensional product manifold $I \times M$ furnished with the metric tensor

$$
\bar{g}=-f^{2} \mathrm{~d} t^{2} \oplus g
$$

is called a standard static space-time and is denoted by $\bar{M}=I_{f} \times M$, where $I$ is an open, connected subinterval of $\mathbb{R}$ and $\mathrm{d} t^{2}$ is the Euclidean metric tensor on $I$. Note that standard static space-times can be considered as a generalization of the Einstein static universe [3-6,13,14].

The following theorem provides a description of the concircular curvature tensor on pseudo-Riemannian warped product manifolds.

Proposition 6 Let $M=M_{1} \times_{f} M_{2}$ be a singly warped product manifold with the metric tensor $g=g_{1} \oplus f^{2} g_{2}$. If $X_{i}, Y_{i}, Z_{i} \in \mathfrak{X}\left(M_{i}\right) i=1,2$, then the concircular curvature tensor $\mathcal{C}$ on $M$ is given by

$$
\mathcal{C}\left(X_{1}, Y_{1}\right) Z_{1}=\mathcal{R}^{1}\left(X_{1}, Y_{1}\right) Z_{1}
$$




$$
\begin{aligned}
& -\frac{\tau}{n(n-1)}\left[g_{1}\left(X_{1}, Z_{1}\right) Y_{1}-g_{1}\left(Y_{1}, Z_{1}\right) X_{1}\right] \\
\mathcal{C}\left(X_{2}, Y_{1}\right) Z_{1}= & {\left[\frac{1}{f} H^{f}\left(Y_{1}, Z_{1}\right)+\frac{\tau}{n(n-1)} g_{1}\left(Y_{1}, Z_{1}\right)\right] X_{2} } \\
\mathcal{C}\left(X_{1}, Y_{2}\right) Z_{2}= & f g_{2}\left(Y_{2}, Z_{2}\right)\left[\nabla_{X_{1}}^{1} \operatorname{grad} f+\frac{\tau f}{n(n-1)} X_{1}\right],
\end{aligned}
$$

and

$$
\begin{aligned}
\mathcal{C}\left(X_{2}, Y_{2}\right) Z_{2}= & \mathcal{R}^{2}\left(X_{2}, Y_{2}\right) Z_{2} \\
& -\left(\|\operatorname{grad} f\|_{1}^{2}+\frac{\tau f^{2}}{n(n-1)}\right)\left[g_{2}\left(X_{2}, Z_{2}\right) Y_{2}-g_{2}\left(Y_{2}, Z_{2}\right) X_{2}\right],
\end{aligned}
$$

where $H^{f}\left(Y_{1}, Z_{1}\right)=g_{1}\left(\nabla_{X_{1}}^{1} \operatorname{grad} f, Z_{1}\right)$ is the Hessian of $f$.

The following theorem is a direct consequence of the above proposition.

Theorem 3 Let $M=M_{1} \times_{f} M_{2}$ be a singly warped product manifold with the metric tensor $g=g_{1} \oplus f^{2} g_{2}$. M is concircularly flat if and only if

(1) $M_{1}$ is of constant curvature

$$
\kappa_{1}=\kappa=\frac{\tau}{n(n-1)} .
$$

(2) $\frac{1}{f} H^{f}\left(Y_{1}, Z_{1}\right)+\frac{\tau}{n(n-1)} g_{1}\left(Y_{1}, Z_{1}\right)=0$, and

(3) $M_{2}$ is of constant curvature

$$
\kappa_{2}=\|\operatorname{grad} f\|_{1}^{2}+\frac{\tau f^{2}}{n(n-1)}=\kappa f^{2}+\|\operatorname{grad} f\|_{1}^{2} .
$$

Now suppose that the concircular curvature tensor $\mathcal{C}$ on $M=M_{1} \times{ }_{f} M_{2}$ vanishes, then equation (4.2) implies that

$$
H^{f}\left(Y_{1}, Z_{1}\right)=\frac{-\tau f}{n(n-1)} g_{1}\left(Y_{1}, Z_{1}\right),
$$

i.e., $M_{1}$ is of Hessian type. Taking the trace of this equation we get that

$$
\Delta f=\frac{-n_{1} \tau}{n(n-1)} f=-n_{1} \kappa_{1} f
$$

Corollary 2 Let $M=M_{1} \times_{f} M_{2}$ be a concircularly flat singly warped product manifold with the metric tensor $g=g_{1} \oplus f^{2} g_{2}$. Then $M_{1}$ is of Hessian type and $\Delta f=-n_{1} \kappa_{1} f$. 
Now, we note that $\mathcal{C}$ can be simplified if the last position is a concurrent field. Let $\zeta=\zeta_{1}+\zeta_{2}$ be a vector field on $M=M_{1} \times_{f} M_{2},\left\{e_{i} \mid 1 \leq i \leq n_{1}\right\}$ be an orthonormal basis of $\mathfrak{X}\left(U_{1}\right)$ and $\left\{e_{i} \mid n_{1}+1 \leq i \leq n_{1}+n_{2}\right\}$ be an orthonormal basis of $\mathfrak{X}\left(U_{2}\right)$ where $U_{i}$ is an open subset of $M_{i}$. Then $\left\{e_{i} \mid 1 \leq i \leq n_{1}+n_{2}\right\}$ is an orthogonal basis of $\mathfrak{X}\left(U_{1} \times{ }_{f} U_{2}\right)$. Thus

$$
\nabla_{e_{i}} \zeta-e_{i}=\nabla_{e_{i}}^{1} \zeta_{1}-e_{i}+e_{i}(\ln f) \zeta_{2}
$$

for $1 \leq i \leq n_{1}$ and

$$
\nabla_{e_{i}} \zeta-e_{i}=\zeta_{1}(\ln f) e_{i}+\nabla_{e_{i}}^{2} \zeta_{2}-f g_{2}\left(\zeta_{2}, e_{i}\right) \operatorname{grad} f-e_{i}
$$

for $n_{1}+1 \leq i \leq n_{1}+n_{2}$.

Lemma 1 Let $M=M_{1} \times_{f} M_{2}$ be a singly warped product manifold with the metric tensor $g=g_{1} \oplus f^{2} g_{2}$. Then $\zeta=\zeta_{1}+\zeta_{2}$ is a concircular vector field on $M=M_{1} \times{ }_{f} M_{2}$ if and only if $\zeta_{1}$ is a concircular vector field on $M_{1}$ and one of the following conditions holds

(1) $\zeta_{2}$ is a concircular vector field on $M_{2}$, and $f$ is constant; or

(2) $\zeta_{2}=0$ and $\zeta_{1}(f)=f$.

Let $\zeta$ be a concurrent vector field, then

$$
\mathcal{R}(X, Y) \zeta=0
$$

Thus

$$
\mathcal{C}(X, Y) \zeta=-\frac{\tau}{n(n-1)}[g(X, \zeta) Y-g(Y, \zeta) X]
$$

Suppose that $M=M_{1} \times{ }_{f} M_{2}$ is a concircularly curvature flat warped product manifold, then

$$
\tau[g(Y, \zeta) X-g(X, \zeta) Y]=0
$$

for any vector fields $X$ and $Y$. Thus $\tau=0$ and consequently $M$ is flat. This discussion leads to the following result.

Theorem 4 Let $M=M_{1} \times{ }_{f} M_{2}$ be a concircularly flat singly warped product manifold with the metric tensor $g=g_{1} \oplus f^{2} g_{2}$. Then $M$ is flat if $M_{1}$ admits a concircular vector field $\zeta_{1}$ and one of the following conditions holds:

(1) $M_{2}$ admits a concircular vector field $\zeta_{2}$ and $f$ is constant; or

(2) $\zeta_{1}(f)=f$.

We will now focus on generalized Robertson-Walker space-times and consider the concircular curvature on this class of space-times by using our previous results. Let 
$\bar{M}=I \times{ }_{f} M$ be a generalized Robertson-Walker space-time equipped with the metric tensor $\bar{g}=-\mathrm{d} t^{2} \oplus f^{2} g$. Then the concircular curvature tensor $\overline{\mathcal{C}}$ on $\bar{M}$ is given by

$$
\begin{aligned}
\overline{\mathcal{C}}\left(\partial_{t}, \partial_{t}\right) \partial_{t} & =0, \overline{\mathcal{C}}\left(X, \partial_{t}\right) \partial_{t}=\frac{-1}{f}\left[\ddot{f}+\frac{\bar{\tau} f}{n(n+1)}\right] X, \\
\overline{\mathcal{C}}\left(\partial_{t}, X\right) Y & =f g(X, Y)\left[\ddot{f}+\frac{\bar{\tau} f}{n(n+1)}\right] \partial_{t}, \\
\overline{\mathcal{C}}(X, Y) Z & =\mathcal{R}(X, Y) Z+\left[\dot{f}^{2}-\frac{\bar{\tau} f^{2}}{n(n+1)}\right][g(X, Z) Y-g(Y, Z) X],
\end{aligned}
$$

for any vector fields $X, Y, Z \in \mathfrak{X}(M)$, where $\mathcal{R}$ is the (Riemann) curvature tensor on $M$. By using direct calculation and our previous results one can conclude the following.

Proposition 7 Let $\bar{M}=I \times_{f} M$ be an $(n+1)$-dimensional generalized RobertsonWalker space-time equipped with the metric tensor $\bar{g}=-\mathrm{d} t^{2} \oplus f^{2} g, n \geq 3$. $\bar{M}$ is concircularly flat if and only if

(1) The scalar curvature of $(\bar{M}, \bar{g})$ satisfies $\ddot{f}+\frac{\bar{\tau} f}{n(n+1)}=0$, and

(2) $(M, g)$ has constant sectional curvature $\kappa \equiv-\left[\dot{f}^{2}+f \ddot{f}\right]$.

The above result gives us a full characterization for the warping function $f$.

Proposition 8 Let $\bar{M}=I \times_{f} M$ be an $(n+1)$-dimensional concircularly flat generalized Robertson-Walker space-time equipped with the metric tensor $\bar{g}=-\mathrm{d} t^{2} \oplus f^{2} g$. Suppose that $\bar{X}=h \partial_{t}+X$ is a vector field on $\bar{M}$, where $X$ is a vector field on $M$ and $h$ is a smooth function on $I$. Then $(\bar{M}, \bar{g})$ is flat if one of the following conditions holds

(1) $M$ admits a concircular vector field and $f$ is constant, or

(2) $f(t)=a t+b$.

Now, we are ready to study concircular curvature tensor $\overline{\mathcal{C}}$ on $\bar{M}={ }_{f} I \times M$. Let $\bar{M}=I_{f} \times M$ be a standard static space-time equipped with the metric tensor $\bar{g}=$ $-f^{2} \mathrm{~d} t^{2} \oplus g$. Then the concircular curvature tensor $\overline{\mathcal{C}}$ on $\bar{M}$ is given by

$$
\begin{aligned}
\overline{\mathcal{C}}\left(X, \partial_{t}\right) \partial_{t} & =-f\left[\nabla_{X} \operatorname{grad} f+\frac{\bar{\tau} f}{n(n+1)} X\right], \\
\overline{\mathcal{C}}\left(\partial_{t}, X\right) Y & =\left[\frac{1}{f} \mathrm{H}^{f}(X, Y)+\frac{\bar{\tau}}{n(n+1)} g(X, Y)\right] \partial_{t}, \\
\overline{\mathcal{C}}(X, Y) Z & =\overline{\mathcal{R}}(X, Y) Z-\frac{\bar{\tau}}{n(n+1)}[g(X, Z) Y-g(Y, Z) X],
\end{aligned}
$$

for any vector fields $X, Y, Z \in \mathfrak{X}(M)$, where $\mathcal{R}$ is the (Riemann) curvature tensor on $M$. Now, we can characterize concircularly flat standard static space-time as:

Proposition 9 Let $\bar{M}=I_{f} \times M$ be an $(n+1)$-dimensional standard static space-time equipped with the metric tensor $\bar{g}=-f^{2} \mathrm{~d} t^{2} \oplus g, n \geq 3 . \bar{M}$ is concircularly flat if and only if 
(1) $\nabla_{X} \operatorname{grad} f=-\frac{\bar{\tau}}{n(n+1)} X$ for any vector field $X$ on $M$, and

(2) $(M, g)$ has constant sectional curvature $\kappa=\frac{\bar{\tau}}{n(n+1)}$.

\section{Concircularly Symmetric Warped Product Manifolds}

A pseudo-Riemannian singly warped product manifold $M$ is said to be concircular symmetric if

$$
\left(\nabla_{\zeta} \mathcal{C}\right)(X, Y, Z)=0
$$

for any vector fields $X, Y, Z$ and $\zeta$. It is clear that (see Sect. 3)

$$
\left(\nabla_{\zeta} \mathcal{R}\right)(X, Y, Z)=0
$$

This condition yields the following consequences

$$
\left(\nabla_{\zeta_{1}} \mathcal{R}\right)\left(X_{1}, Y_{1}, Z_{1}\right)=\left(\nabla_{\zeta_{1}}^{1} \mathcal{R}^{1}\right)\left(X_{1}, Y_{1}, Z_{1}\right)=0
$$

Thus $M_{1}$ is locally symmetric. The second case is

$$
\left(\nabla_{\zeta_{1}} \mathcal{R}\right)\left(X_{2}, Y_{1}, Z_{1}\right)=0
$$

This yields

$$
\begin{aligned}
- & \frac{1}{f^{2}} \zeta_{1}(f) H^{f}\left(Y_{1}, Z_{1}\right) X_{2} \\
& +\frac{1}{f} g_{1}\left(\nabla_{\zeta_{1}}^{1} \nabla_{Y_{1}}^{1} \operatorname{grad} f, Z_{1}\right) X_{2}-\frac{1}{f} H^{f}\left(\nabla_{\zeta_{1}}^{1} Y_{1}, Z_{1}\right) X_{2}=0
\end{aligned}
$$

i.e., $\mathcal{F}=\frac{1}{f} H^{f}$ is parallel. The next case is

$$
\begin{aligned}
\left(\nabla_{\zeta_{2}} \mathcal{R}\right)\left(X_{2}, Y_{1}, Z_{1}\right) & =0 \\
0 & =\nabla_{\zeta_{2}} \mathcal{R}\left(X_{2}, Y_{1}\right) Z_{1}-\mathcal{R}\left(\nabla_{\zeta_{2}} X_{2}, Y_{1}\right) Z_{1}-Z_{1}(\ln f) \mathcal{R}\left(X_{2}, Y_{1}\right) \zeta_{2} \\
& =\mathcal{F}\left(Z_{1}, Y_{1}\right) \nabla_{\zeta_{2}} X_{2}-\mathcal{R}\left(\nabla_{\zeta_{2}} X_{2}, Y_{1}\right) Z_{1}+Z_{1}(f) g_{2}\left(X_{2}, \zeta_{2}\right) \nabla_{Y_{1}}^{1} \operatorname{grad} f
\end{aligned}
$$

and so

$$
\mathcal{R}^{1}\left(\operatorname{grad} f, Y_{1}\right) Z_{1}=\mathcal{F}\left(Z_{1}, Y_{1}\right) \operatorname{grad} f-Z_{1}(\ln f) \nabla_{Y_{1}}^{1} \operatorname{grad} f
$$

Now, we have

$$
\left(\nabla_{\zeta_{1}} \mathcal{R}\right)\left(X_{2}, Y_{2}, Z_{2}\right)=\left(\nabla_{\zeta_{2}} \mathcal{R}\right)\left(X_{1}, Y_{2}, Z_{2}\right)=0
$$


Thus

$$
X_{1}(f) \mathcal{R}^{2}\left(\zeta_{2}, Y_{2}\right) Z_{2}=\left(X_{1}(f)\|\operatorname{grad} f\|^{2}-f^{2} \mathcal{F}\left(X_{1}, \operatorname{grad} f\right)\right) G_{2}\left(\zeta_{2}, Y_{2}, Z_{2}\right),
$$

where $G_{2}\left(\zeta_{2}, Y_{2}, Z_{2}\right)=\left[g_{2}\left(\zeta_{2}, Z_{2}\right) Y_{2}-g_{2}\left(Y_{2}, Z_{2}\right) \zeta_{2}\right]$. The next case is

$$
\left(\nabla_{\zeta_{2}} \mathcal{R}\right)\left(X_{2}, Y_{2}, Z_{2}\right)=0
$$

This yields

$$
\left(\nabla_{\zeta_{2}}^{2} \mathcal{R}^{2}\right)\left(X_{2}, Y_{2}, Z_{2}\right)=0
$$

Theorem 5 Let $M=M_{1} \times_{f} M_{2}$ be a concircularly symmetric warped product manifold with the metric tensor $g=g_{1} \oplus f^{2} g_{2}$. Then,

(1) both $M_{1}$ and $M_{2}$ are locally symmetric,

(2) $M_{2}$ is of constant curvature given that $f$ is not constant, and

(3) $\mathcal{F}=\frac{1}{f} H^{f}$ is parallel.

\section{Divergence-free Concircular Curvature Tensor}

It is well known that the Riemann tensor is harmonic if and only if the Ricci tensor is a Codazzi tensor, i.e., for any vector fields $X, Y, Z \in \mathfrak{X}(M)$, we have

$$
\left(\nabla_{X} \operatorname{Ric}\right)(Y, Z)=\left(\nabla_{Y} \operatorname{Ric}\right)(X, Z)
$$

Moreover, the concircular curvature tensor is divergence free if and only if the Riemann tensor is harmonic. Let us define

$$
T(X, Y, Z)=\left(\nabla_{X} \operatorname{Ric}\right)(Y, Z)-\left(D_{Y} \operatorname{Ric}\right)(X, Z)
$$

for any vector fields $X, Y, Z \in \mathfrak{X}(M)$. It is clear that the Ricci tensor is a Codazzi tensor if and only if $T(X, Y, Z)$ vanishes. Let $\left(M_{1} \times_{f} M_{2}, g\right)$ be a singly warped product manifold with $T(X, Y, Z)=0$. Then

$$
\begin{aligned}
T^{1}\left(X_{1}, Y_{1}, Z_{1}\right)= & \frac{n_{2}}{f} Y_{1}(f) \mathcal{F}\left(X_{1}, Z_{1}\right)-\frac{n_{2}}{f} X_{1}(f) \mathcal{F}\left(Y_{1}, Z_{1}\right) \\
& -\frac{n_{2}}{f} \mathcal{R}^{1}\left(X_{1}, Y_{1}, \operatorname{grad} f, Z_{1}\right) .
\end{aligned}
$$

The next case is

$$
\begin{aligned}
0= & X_{1}\left(f^{\sharp}\right) g_{2}\left(Y_{2}, Z_{2}\right)-2 X_{1}(\ln f) \operatorname{Ric}\left(Y_{2}, Z_{2}\right) \\
& -Y_{1}\left(f^{\sharp}\right) g_{2}\left(X_{2}, Z_{2}\right)+2 Y_{1}(\ln f) \operatorname{Ric}\left(X_{2}, Z_{2}\right)
\end{aligned}
$$




$$
X_{1}(f) \operatorname{Ric}\left(Y_{2}, Z_{2}\right)=f\left(X_{1}\left(f^{\sharp}\right)-f \operatorname{Ric}\left(X_{1}, \operatorname{grad} f\right)\right) g_{2}\left(Y_{2}, Z_{2}\right) .
$$

Finally,

$$
T^{2}\left(X_{2}, Y_{2}, Z_{2}\right)=0
$$

The tensor $T$ vanishes in the rest cases. Now, one can write the following results.

Theorem 6 Let $\left(M_{1} \times_{f} M_{2}, g\right)$ be a singly warped product manifold with warping function $f>0$ on $M_{1}$. Assume the concircular curvature tensor $\mathcal{C}$ is divergence free. Then,

(1) the concircular curvature tensor $\mathcal{C}^{1}$ is divergence free if

$$
\mathcal{R}^{1}\left(X_{1}, Y_{1}, \operatorname{grad} f, Z_{1}\right)=Y_{1}(f) \mathcal{F}\left(X_{1}, Z_{1}\right)-X_{1}(f) \mathcal{F}\left(Y_{1}, Z_{1}\right)
$$

(2) the concircular curvature tensor $\mathcal{C}^{2}$ is divergence free, and

(3) $f$ is constant or $\left(M_{2}, g_{2}\right)$ is Einstein.

Theorem 7 Let $\left(M_{1} \times_{f} M_{2}, g\right)$ be a singly warped product manifold with warping function $f>0$ on $M_{1}$. The concircular curvature tensor of the metric tensor $g$ is divergence free if

(1) $f$ is constant and the concircular curvature tensors $\mathcal{C}^{i}$ of the metric tensors $g_{i} ; i=$ 1, 2 are divergence free, or

(2) $H^{f}=0, \mathcal{C}^{1}$ is divergence free and $\left(M_{2}, g_{2}\right)$ is Einstein with factor $g_{1}(\operatorname{grad} f$, $\operatorname{grad} f)$.

The following results are special cases on a generalized Robertson-Walker spacetime and on a standard static space-time.

Corollary 3 Let $\bar{M}=I \times_{f} M$ be a generalized Robertson-Walker space-time with the metric tensor $\bar{g}=-\mathrm{d} t^{2} \oplus f^{2}$. If the concircular curvature tensor $\overline{\mathcal{C}}$ of $(\bar{M}, \bar{g})$ is divergence free, then the concircular curvature tensor $\mathcal{C}$ of $(M, g)$ is divergence free. If, in addition, $f=a t+b$, then $(M, g)$ is Einstein.

Corollary 4 Let $\bar{M}=I \times{ }_{f} M$ be a generalized Robertson-Walker space-time with the metric tensor $\bar{g}=-\mathrm{d} t^{2} \oplus f^{2} g$. Then the concircular curvature tensor $\overline{\mathcal{C}}$ of $(\bar{M}, \bar{g})$ is divergence free if

(1) $f$ is constant and the concircular curvature tensor $\mathcal{C}$ of $(M, g)$ is divergence free, or

(2) $f=a t+b$ and $(M, g)$ is Einstein with factor $-a^{2}$.

Corollary 5 Let $\bar{M}={ }_{f} I \times M$ be a standard static space-time with the metric tensor $\bar{g}=-f^{2} \mathrm{~d} t^{2} \oplus g$ and $H^{f}=0$. Then the concircular curvature tensor $\overline{\mathcal{C}}$ of $(\bar{M}, \bar{g})$ is divergence free if and only if the concircular curvature tensor $\mathcal{C}$ of $(M, g)$ is divergence free. 
Acknowledgements We would like to thank the referees for their careful reviews and valuable comments which helped us to improve quality of the paper.

\section{References}

1. Ahsan, Z.: Tensors: Mathematics of Differential Geometry and Relativity. PHI Learning Pvt. Ltd., Delhi (2017). (Second Printing December 2017)

2. Ahsan, Z., Siddiqui, S.A.: Concircular curvature tensor and fluid space-times. Int. J. Theor. Phys. 48, 3202-3212 (2009)

3. Allison, D.E.: Geodesic completeness in static space-times. Geom. Dedic. 26, 85-97 (1988)

4. Allison, D.E.: Energy conditions in standard static space-times. Gen. Relat. Gravit. 20(2), 115-122 (1998)

5. Allison, D.E., Ünal, B.: Geodesic completeness in static space-time. J. Geom. Phys. 46(2), 193-200 (2003)

6. Besse, A.L.: Einstein Manifolds, Classics in Mathematics. Springer, Berlin (2008)

7. Bishop, R.L., O’Neill, B.: Manifolds of negative curvature. Trans. Am. Math. Soc. 145, 1-49 (1969)

8. Boeckx, E.: Einstein like semi symmetric spaces. Arch. Math., Tomus 29, 235-240 (1992)

9. Catino, G., Mantegazza, C., MazzieriL, L.: A note on Codazzi tensors. Math. Annalen 362(1-2), 629-638 (2015)

10. Chen, B.-Y.: Some results on concircular vector fields and their applications to Ricci solitons. Bull. Korean Math. Soc. 52(5), 1535-1547 (2015)

11. El-Sayied, H.K., Shenawy, S., Syied, N.: Conformal vector fields on doubly warped product manifolds and applications. Adv. Math. Phys. 11, Article ID 6508309 (2016)

12. El-Sayied, H.K., Sameh, S., Syied, N.: On symmetries of generalized Robertson-Walker spacetimes and applications. J. Dyn. Sys. Geom. Theor. 15(1), 51-69 (2017)

13. El-Sayied, H.K., Shenawy, S., Syied, N.: Symmetries of $f$-associated standard static spacetimes and applications. J. Egypt. Math. Soc. 25(4), 414-418 (2017)

14. El-Sayied, H.K., Shenawy, S., Syied, N.: Locally symmetric $f$-associated standard static spacetimes. Math. Methods Appl. Sci 41(15), 5733-5736 (2018)

15. Flores, J.L., Sánchez, M.: Geodesic connectedness and conjugate points in GRW spacetimes. J. Geom. Phys. 36(3-4), 285-314 (2000)

16. Gouli-Andreou, F., Moutafi, E.: On the concircular curvature of a $(k, \mu, v)$-manifold. Pac. J. Math. 269(1), 113-132 (2014)

17. Kim, In-Bae: Special concircular vector fields in Riemannian manifolds. Hiroshima Math. J. 12(1), 77-91 (1982)

18. Majhi, P., De, U.C.: Concircular curvature tensor on $K$-contact manifolds. Acta Mathematica Academiae Paedagogicae Nyregyhaziensis 29, 89-99 (2013)

19. Majhi, P., De, U.C.: Classifications of $N(k)$-contact metric manifolds satisfying certain curvature conditions. Acta Math. Univ. Comenianae LXXXIV(1), 167-178 (2015)

20. O’Neill, B.: Semi-Riemannian Geometry with Applications to Relativity. Academic Press Limited, London (1983)

21. Olszak, K., Olszak, Z.: On pseudo-Riemannian manifolds with recurrent concircular curvature tensor. Acta Math. Hangarica 137, 64-71 (2012)

22. Pokhariyal, G.P.: Curvature tensors in Riemannian manifolds II. Proc. Math. Sci. 79(3), 105-110 (1974)

23. Pokhariyal, G.P.: Relativistic significance of curvature tensors. Int. J. Math. Math. Sci. 5(1), 133-139 (1982)

24. Pokhariyal, G.P., Mishra, R.S.: Curvature tensors in Riemannian manifolds. Indian J. Pure Appl. Math. 2(3), 529-530 (1970)

25. Pokhariyal, G.P., Mishra, R.S.: Curvature tensors and their relativistics significance. Yokohama Math. J. 18, 105-108 (1970)

26. Sánchez, M.: On the geometry of generalized Robertson-Walker spacetimes: curvature and killing fields. J. Geom. Phys. 31(1), 1-15 (1999)

27. Sánchez, M.: On the geometry of generalized Robertson-Walker spacetimes: geodesics. Gen. Relat. Gravit. 30(6), 915-932 (1998) 
28. Singh, H., Khan, Q.: On symmetric Riemannian manifolds. Novi Sad J. Math. 29(3), 301-308 (1999)

29. Shenawy, S., Ünal, B.: 2-Killing vector fields on warped product manifolds. Int. J. Math. 26, 1550065 (2015)

30. Shenawy, S., Ünal, B.: The $W_{2}$-curvature tensor on warped product manifolds and applications. Int. J. Geom. Methods Mod. Phys. 13(7), 1650099 (2016)

31. Yano, K.: Concircular geometry I. Concircular transformations. Proc. Imp. Acad. 16(6), 195-200 (1940)

32. Yano, K., Kon, M.: Structures on Manifolds. World Scientific Publishing, Singapore (1984)

33. Youssef, N.L., Soleiman, A.: On concircularly recurrent Finsler manifolds. Balk. J. Geom. Appl. 18(1), 101-113 (2013)

34. Zlatanovica, M., Hinterleitnerb, I., Najdanovi, M.: On equitorsion concircular tensors of generalized Riemannian spaces. Filomat 28(3), 463-471 (2014)

Publisher's Note Springer Nature remains neutral with regard to jurisdictional claims in published maps and institutional affiliations. 